Laser Chem.

Volume 1(1), October 1982, pp. 17-36

0278-6273/82/0101-0017\$6.50/0

(C) 1982 harwood academic publishers gmbh

Printed in the United States of America

\title{
Spectral and Temperature Dependence of Laser Induced Dissociative Attachment in $\mathbf{S F}_{\mathbf{6}}$
}

\author{
R. BARBE, J.-P. ASTRUC, A. LAGREZE and J.-P. SCHERMANN \\ Laboratoire de Physique des Lasers, Associé au C.N.R.S. n`282 Centre \\ Scientifique et Polytechnique, Université Paris-Nord - 93430 - Villetaneuse, \\ France
}

(Received March 12, 1982; in final form May 6, 1982)

\begin{abstract}
We consider the influence of resonant laser excitation upon the dissociative reaction between $\mathrm{SF}_{6}$ molecules and very low energy electrons. Experimentally, the use of valence electrons of atoms in Rydberg states ensures a well-defined energy resolution of the scattering electrons in the crossed beam apparatus. A spectroscopic model of the infrared absorption of $\mathrm{CO}_{2}$ laser radiation by $\mathrm{SF}_{6}$ gives a fair determination of the energy levels and vibrational transitions involved in the dissociative process. This reaction between highly excited atoms and a molecule is interpreted as a three step process: attachment of a quasi-free electron, followed by the interaction between the atomic ionic core and the negative molecular ion leading to a partial stabilization and, finally, the competition between the dissociating and the autodetachment channels.
\end{abstract}

\section{INTRODUCTION}

The multiple photon dissociation (MPD) of $\mathrm{SF}_{6}$ into neutral components occurs in two stages, ${ }^{1}$ one involving a resonant excitation of the first few discrete vibrational states of the $v_{3}$ ladder, the other leading to dissociation by absorption of a large number of photons within the vibrational quasicontinuum.

An alternative approach to isotopically selective dissociation of $\mathrm{SF}_{6}$ has been proposed and studied by several authors. ${ }^{2-4}$ In this approach, one takes advantage of a well-known property of $\mathrm{SF}_{6}$ : it attaches low energy electrons and, according to its vibrational energy, the negative ion produced 
is either $\mathrm{SF}_{6}^{-}$or $\mathrm{SF}_{5}^{-}$. Thus, the resonant vibrational excitation can strongly modify the branching ratio between the non-dissociative and dissociative channels [Figure 1]. The large amount of nonresonant laser energy which is required in the second step of the MPD dissociation into neutral components is replaced by chemical energy, i.e. the electron affinity of the $\mathrm{SF}_{5}$ product.

A modeling of the above processes requires a good knowledge of the collision conditions (for instance, single collision conditions), of the $\mathrm{SF}_{6}$ internal energy and the electron energy distribution. Reciprocally, such a model provides information about the dynamics of low energy electron$\mathrm{SF}_{6}$ scattering (attachment, autodetachment and dissociation lifetimes . . . ) and parameters of laser excitation.

We report here the spectral and temperature dependence of the following reactions:

$$
\mathrm{SF}_{6}^{*}+\mathrm{e} \rightarrow \mathrm{SF}_{6}^{-} \overbrace{\mathrm{SF}_{5}^{-}+\mathrm{F}}^{\mathrm{SF}_{6}+\mathrm{e}}
$$

where $\mathrm{SF}_{6}\left(E_{v}\right)$ corresponds to $\mathrm{SF}_{6}$ molecules with an internal energy $E_{v}$ controlled by means of temperature and $\mathrm{SF}_{6}^{*}$ to laser excited molecules. In order to obtain a knowledge of the electron energy distribution as accurately as possible, we use valence electrons of atoms in Rydberg states. Experimentally, this way of producing sub-thermal electrons is somewhat similar to the use of a photoionisation source of quasi monoenergetic electrons. ${ }^{5}$ In comparison with more conventional techniques ${ }^{6}$ such as RPD spectrometers or swarm experiments in which the electron energy distributions are approximately $100 \mathrm{meV}$ wide with long energy tails, the use of electrons bound to highly excited atoms sets an upper limit to the maximum kinetic energy of the electrons attaching to $\mathrm{SF}_{6}$. As far as attachment alone is concerned, it has been shown by Matsuzawa ${ }^{7}$ that these bound electrons behave as if they were free, with momentum distributions governed by the quantum states of the highly excited atoms. The fate of the negative ion created by this charge exchange may, however, be influenced by the presence of the remaining ionic core, leading to a stabilization process. ${ }^{8}$

For a wide range of temperature and, thus, of internal energy of the 


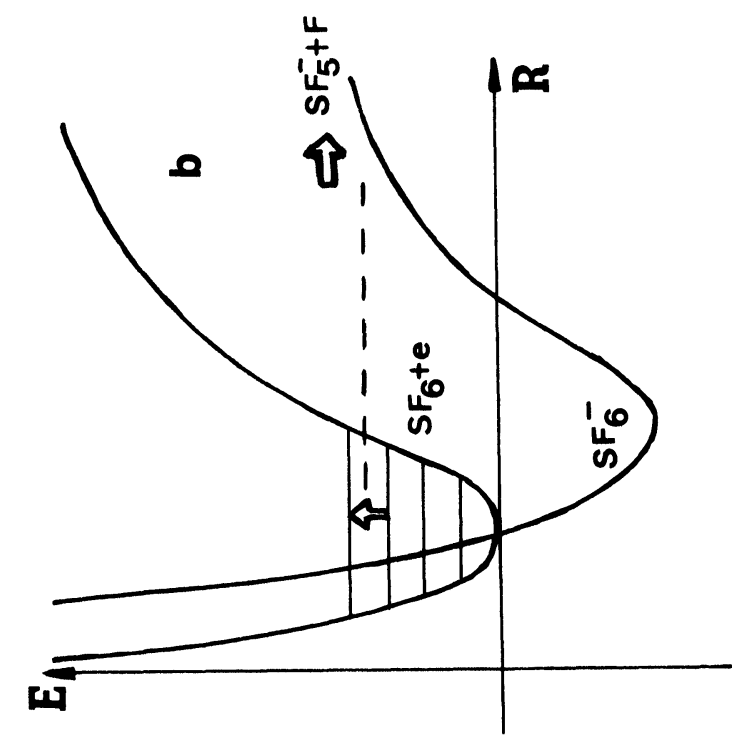

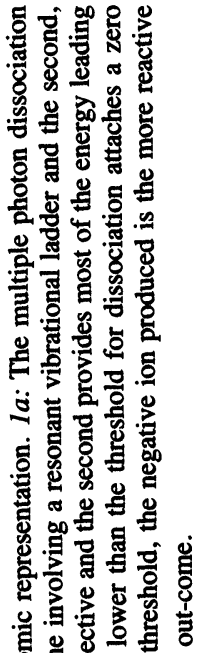

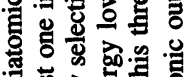

总密要

.

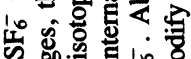

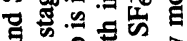

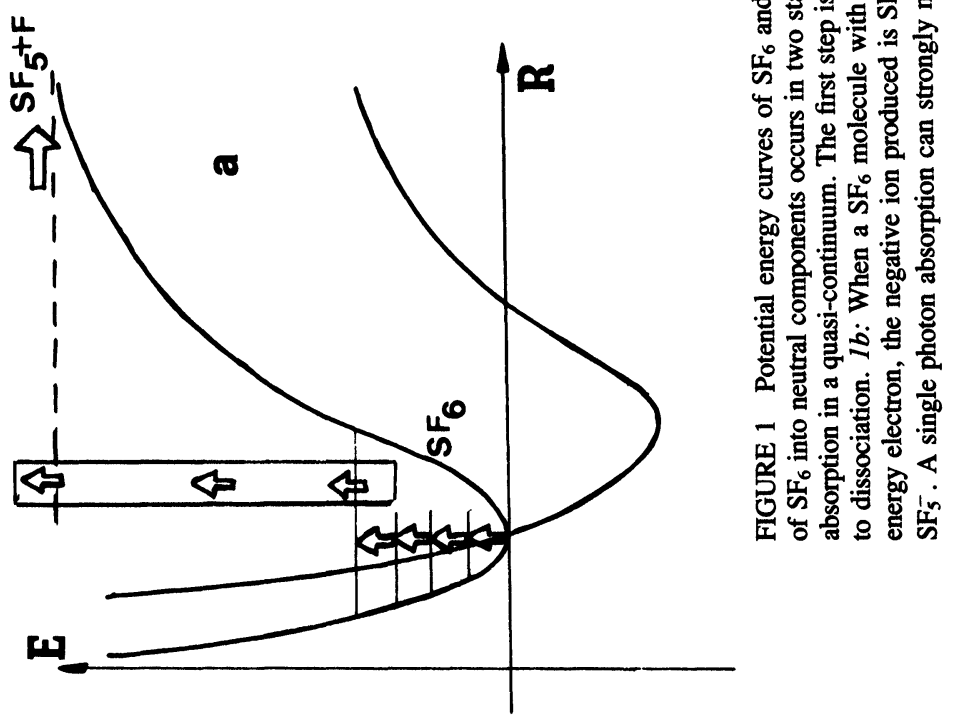


parent $\mathrm{SF}_{6}$ molecules, we measured the influence of resonant laser excitation upon the reaction (1) for all $\mathrm{CO}_{2}$ laser lines corresponding to the absorption of the isotopic molecule ${ }^{32} \mathrm{SF}_{6}$. Our results agree reasonably well with those of Chen and Chantry ${ }^{3}$ in respect of the spectral dependence of the process. Their experiment and interpretation concerning the laser excitation and energy threshold were in disagreement with previous measurements of the activation energy of the reaction. We attribute this disagreement to the problem of electron energy distributions in RPD techniques.

By analyzing our results with a spectroscopic model of $\mathrm{CO}_{2}$ laser absorption by $\mathrm{SF}_{6}$, derived from the model of Nowack and Lyman, ${ }^{9}$ we interpret the red shift of the dissociative channel with respect to the low temperature small signal absorption curves and we show that single photon excitation is responsible for the laser induced process. From our data, we confirm the accepted value of energy threshold of the reaction (1) and show that, following the suggestion of Nowack and Lyman, one must add to their model the variation of the rotational constant as a function of vibrational energy. We also demonstrate that one has to take into account, for high excitation levels, the competition between the two exit channels of reaction (1), i.e., dissociative attachment and autodetachment.

\section{APPARATUS}

A schematic diagram of the experimental arrangement is shown in Figure 2. This cross beam experiment, which satisfies the single collision condition, will be described in more detail in a forthcoming paper. ${ }^{10} \mathrm{~A}$ beam of $\mathrm{SF}_{6}$ molecules, emanating from a platinum tube $(1 \mathrm{~mm}$ i.d. $10 \mathrm{~cm}$ long) with variable temperature from $300^{\circ} \mathrm{K}$ to $1000^{\circ} \mathrm{K}$ crosses a beam of argon atoms excited to Rydberg states. These highly excited atoms which act as a source of low energy electrons, are produced by electron bombardment. Their time of flight between their creation and the collision region is approximately $200 \mu \mathrm{s}$. The competition between the cross-section for creation of these atoms in the state of a principal quantum number $\boldsymbol{n}$ (low orbital quantum number $l$ ) which varies as $n^{-3}$, and the radiative lifetimes which vary as $n^{\alpha}$ where $4<\alpha<5$ (for large values of $l$ after redistribution among $l$ by collisions undergone after creation) results in an energy distribution of the electrons between 10 and $40 \mathrm{meV}(18<n<35)$ as confirmed by field ionization measurements. ${ }^{10}$ 


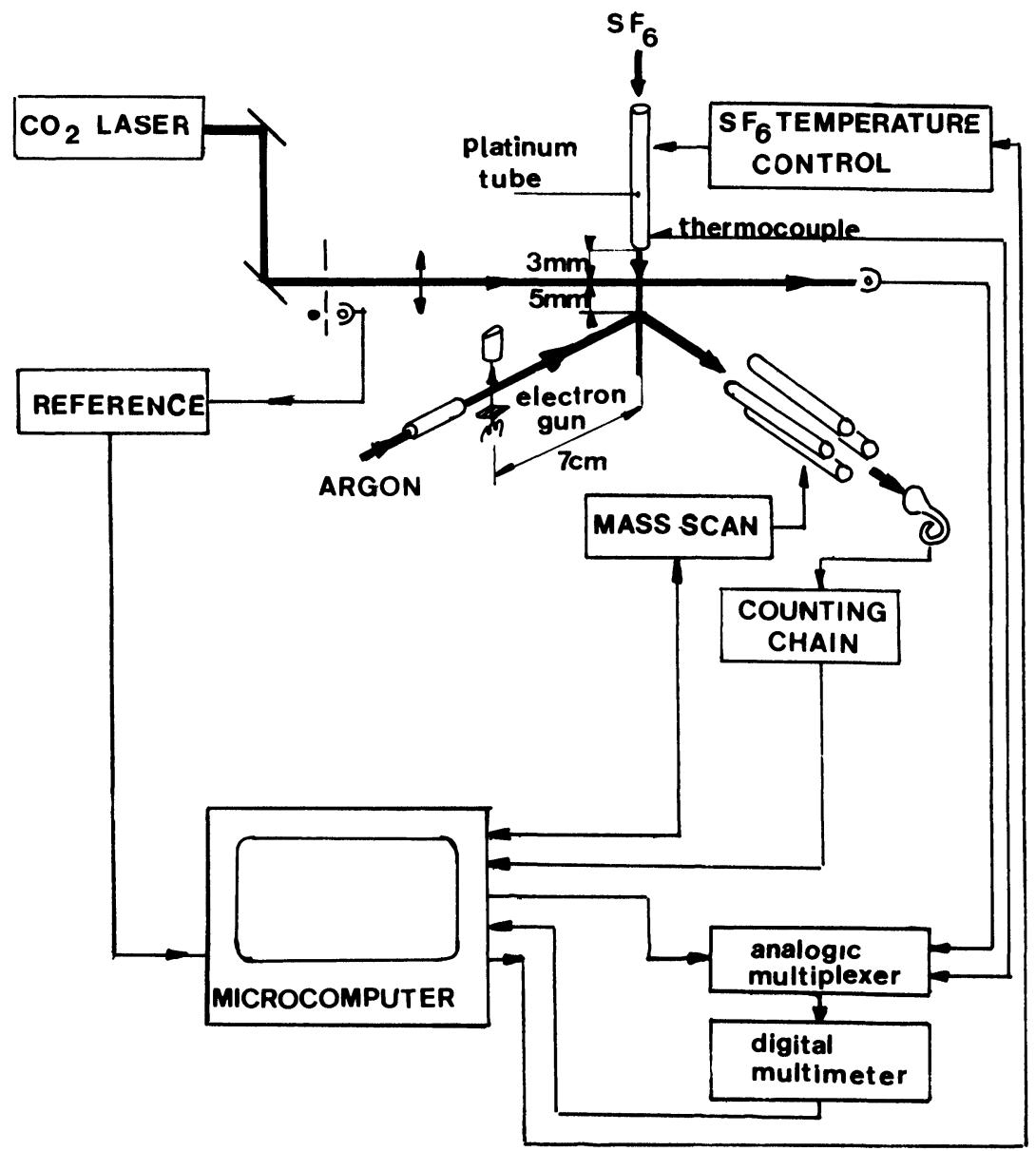

FIGURE 2 Experimental set-up. The $\mathrm{SF}_{6}$ beam with computer controlled temperature (varying between $300^{\circ} \mathrm{K}$ and $1000^{\circ} \mathrm{K}$ ), is excited by the $\mathrm{CO}_{2}$ laser beam. The chopping reference drives the up-counting and down-counting in the micro-computer. The laser excited $\mathrm{SF}_{6}$ beam crosses the high Rydberg argon atomic beam and the ionic products are mass-analyzed by a quadrupole-spectrometer.

A CW $4 \mathrm{~W} \mathrm{CO}$ laser, which is grating tuned, is focused at the exit of the platinum tube and a fraction of the $\mathrm{SF}_{6}^{*}$ beam is excited in the $.8 \mathrm{~mm}$ diameter laser waist. In order to monitor the number of $\mathrm{SF}_{5}^{-}$ions (detected by a quadrupole mass analyzer) produced, due to laser vibrational excitation, ON - OFF synchronous detection is done by a microcomputer 


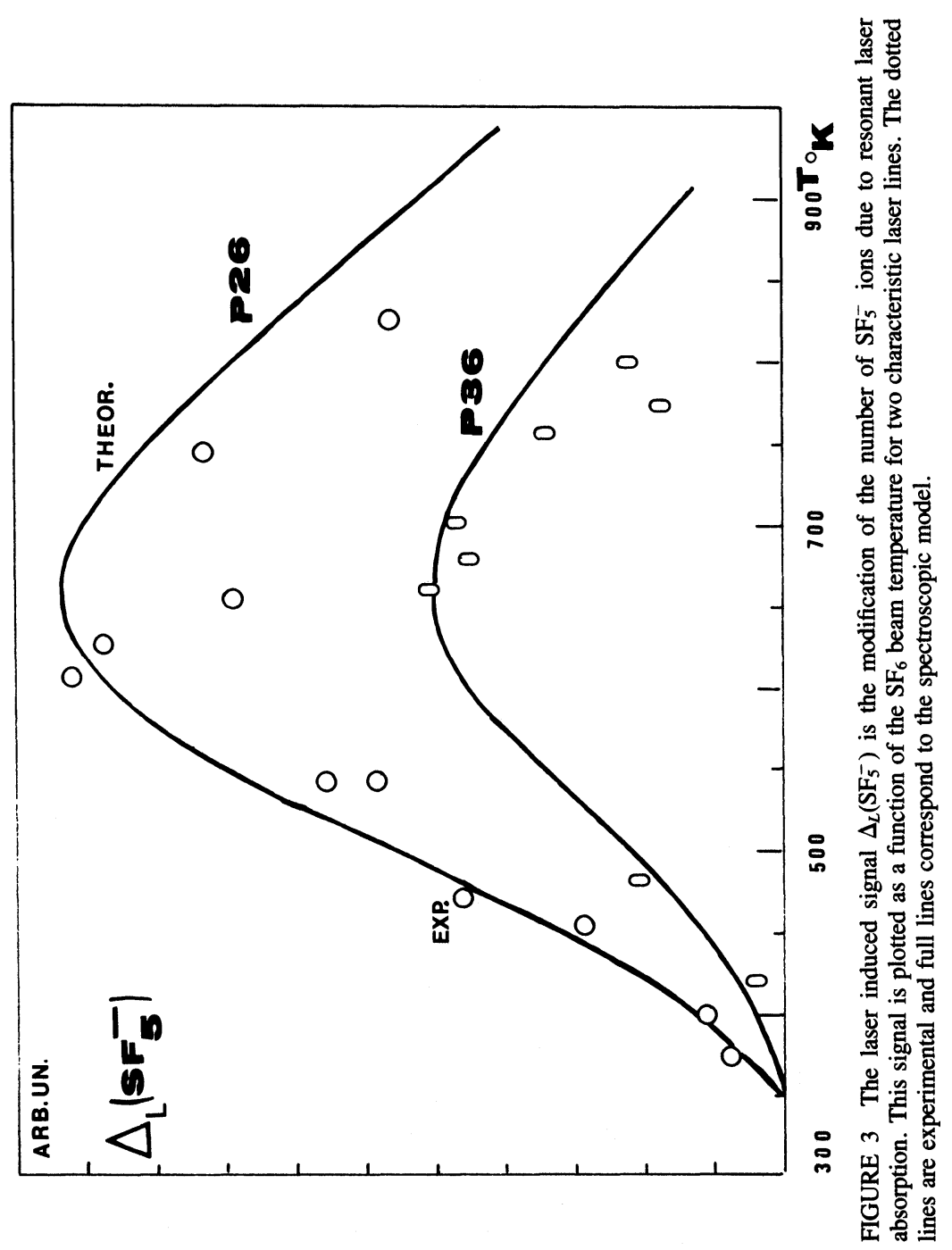


clocked by the laser chopper. Typical ${ }^{32} \mathrm{SF}_{5}^{-}$ion signals due to the laser are 15 counts/sec. with an averaging time of $60 \mathrm{sec}$. The weakness of these signals, due to the crossing beam intensities, did not allow us to observe ${ }^{34} \mathrm{SF}_{5}^{-}$ion signals, restricting our study to the most abundant isotope ${ }^{32} \mathrm{SF}_{6}$ (95\%).

\section{Experimental results}

For several $\mathrm{CO}_{2}$ laser lines corresponding to the absorption band of ${ }^{32} \mathrm{SF}_{6}$, the relative enhancement of the $\mathrm{SF}_{6}^{-} \rightarrow \mathrm{SF}_{5}^{-}+\mathrm{F}$ dissociative channel, as a function of the $\mathrm{SF}_{6}$ parent molecule temperature, was measured (Figure $3)$. The qualitative behavior is the same for the different laser lines: the laser induced $\mathrm{SF}_{5}^{-}$signal rises around $400^{\circ} \mathrm{K}$, culminates near $600^{\circ} \mathrm{K}$ and decreases at higher temperatures.

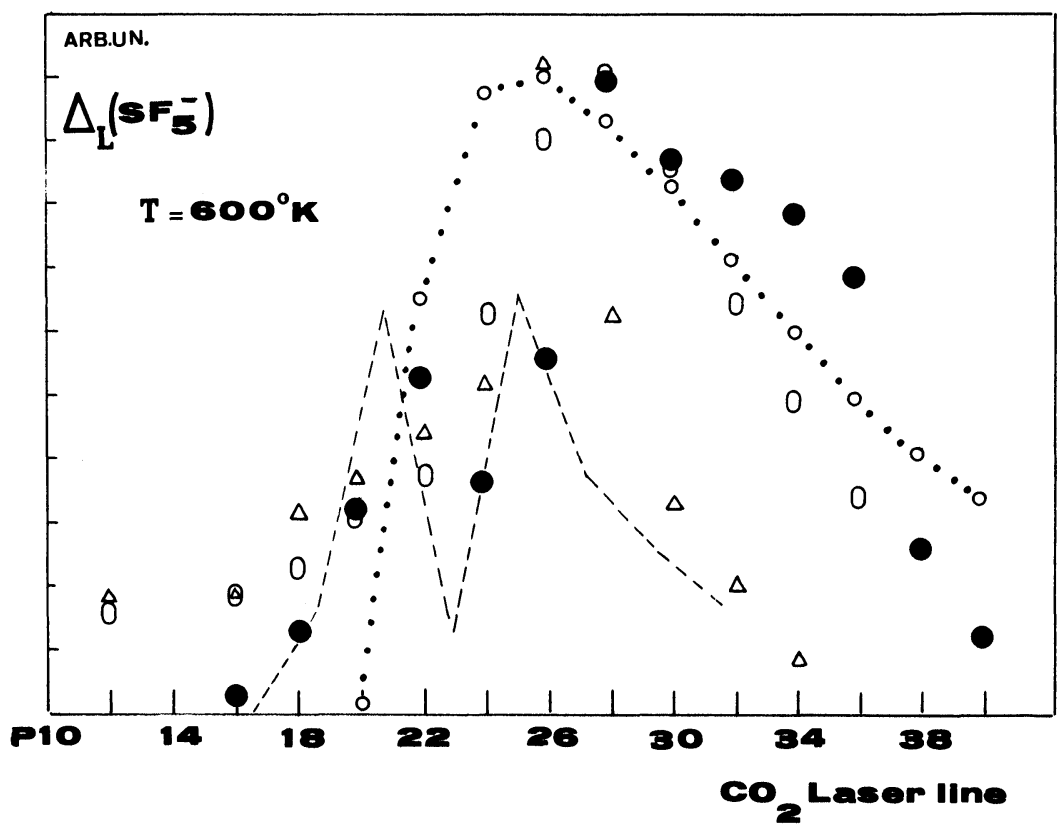

FIGURE 4 The laser induced signal is plotted as a function of the $\mathrm{CO}_{2}$ laser lines for several experiments 0 Chen and Chantry (Ref. 3) $\Delta$ Avrillier and Schermann (Ref. 2) and $\bullet$ this experiment. The dotted line corresponds to the spectroscopic model with a new value of the anharmonic constants and variation of the rotation constant. The broken line corresponds to the original model of Ref. 9. 
At a temperature of $600^{\circ} \mathrm{K}$ at which the laser induced $\mathrm{SF}_{5}^{-}$signal is maximum, the dependence of this signal upon the $\mathrm{CO}_{2}$ laser lines is shown in Figure 4. This measurement agrees reasonably well with that of Chen and Chantry ${ }^{3}$ obtained with an RPD technique and that of Avrillier and Schermann ${ }^{2}$ obtained by an optogalvanic detection in a $\mathrm{SF}_{6}$ discharge.

The power dependence of the laser induced signal is shown in Figure 5. The maximum power available corresponds to a power broadening of about $70 \mathrm{MHz}$ as compared with the $30 \mathrm{MHz}$ Doppler linewidth. In the vibrational excitation region that we consider where $E_{v} \sim 3500 \mathrm{~cm}^{-1}$, there are approximately $10^{2}$ vibrational levels per $\mathrm{cm}^{-1}$ and, thus, one level every $300 \mathrm{MHz}$ which may absorb. This interval is larger than the laser broadening.

The interaction time of the $\mathrm{SF}_{6}$ molecules inside the laser beam waist is $2 \mu \mathrm{s}$. With a $\mathrm{CO}_{2}$ laser photon absorption cross-section of $10^{-18} \mathrm{~cm}^{2}$,

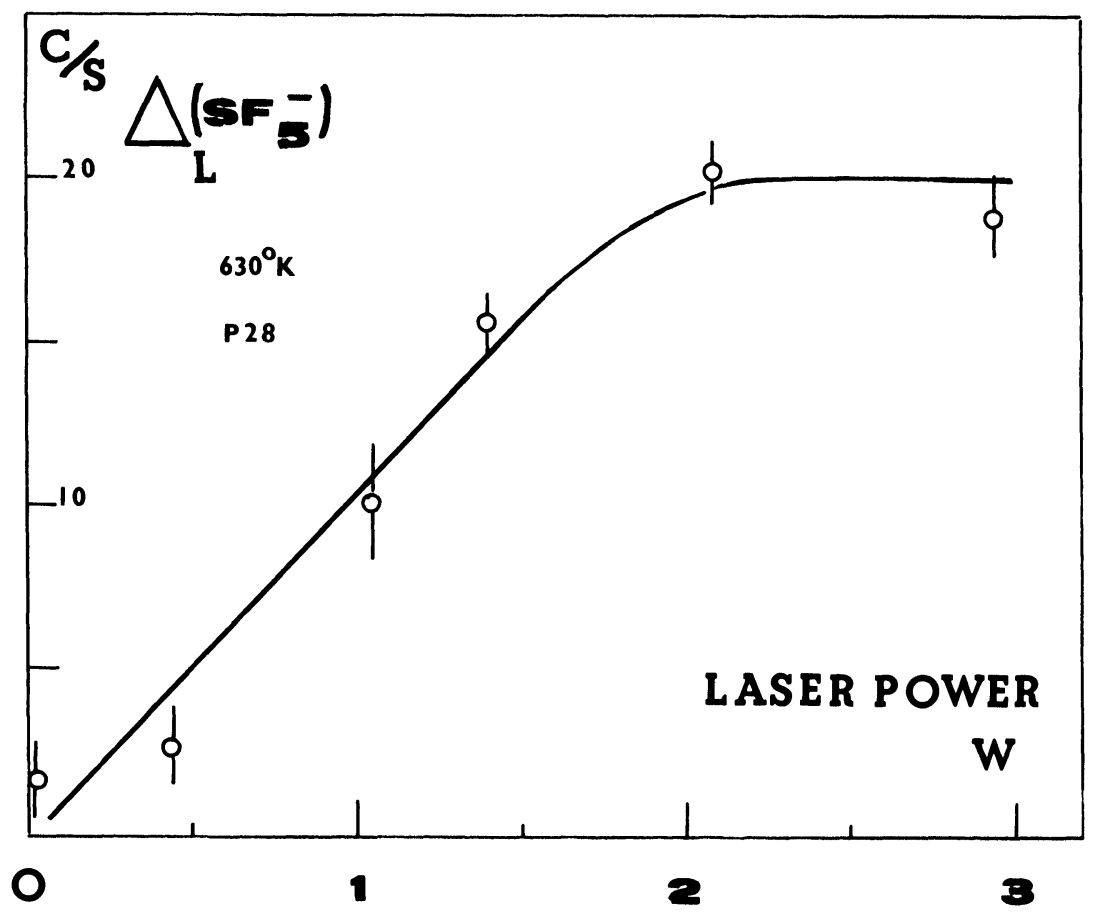

FIGURE 5 The laser induced signal is plotted, as a function of the $\mathrm{CO}_{2}$ laser power for $\mathrm{SF}_{6}$ temperature of $630^{\circ} \mathrm{K}$ and the $\mathrm{P} 28 \mathrm{CO}_{2}$ laser line for which this signal is maximum. 
saturation is obtained for a few $\mathrm{kW} / \mathrm{cm}^{2}$. We thus interpret the power dependence as a linear variation followed by saturation when the probability of excitation of a $\mathrm{SF}_{6}$ molecule reaches unity.

\section{Spectroscoplc model}

The modeling of the first discrete multiple photon stage of excitation in MPD of $\mathrm{SF}_{6}$ has provided a strong impetus for the determination of the infrared spectrum of this molecule. Till now, a precise interpretation of the very high resolution spectrum has only been made for the $\nu_{3}$ fundamental transition, ${ }^{11}$ together with a Doppler limited analysis ${ }^{12}$ of the $\nu_{3} \rightarrow$ $2 v_{3}$ second harmonic and a determination ${ }^{13}$ of the $v_{3}$ vibrational ladder. Very little information is available about the rovibrational spectrum of the other modes, even at low resolution. We have thus used for our spectral analysis the model of Nowack and Lyman ${ }^{9}$ with some modification of the anharmonicity constant, recently determined, ${ }^{13}$ and the energy dependence of the rotational constant which was necessary to introduce in order to fit our data to the initial values of Ref. 9. We shall give here only a brief account of this model referring to the original paper.

The vibrational states of $\mathrm{SF}_{6}$ are defined by a set of vibrational quantum numbers $\left[v_{i}\right]$ corresponding to the 6 normal modes. The only anharmonicity constant which has been precisely measured corresponds to the $\nu_{3}$ mode and we adopt the empirical Nowack and Lyman proposal which states that, for each mode, the harmonic shift of a vibrational overtone is proportional to the frequency $v_{i}$ of the fundamental. Ignoring rotation energy, the frequency of a transition involving a $v_{3}$ photon, originating from a state $\left[v_{i}\right]$ is then given by:

$$
v_{o}\left[v_{i}\right]=v_{o}(0)+A \sum_{i} v_{i} v_{i}
$$

where $v_{o}(0)$ is the frequency of the $\nu_{3}$ transition originating from the ground state. This unique proportionality constant $A$ is related to the anharmonicity constant $X_{33}$ by the relation $X_{33}=\frac{1}{2} A v_{3}$. We take for $X_{33}$ the experimental value of Ref. $13 X_{33}=-1.74 \mathrm{~cm}^{-1}$.

For vibrational energies $E_{v}$ of states $\left[v_{i}\right]$ ranging from 0 to $1650 \mathrm{~cm}^{-1}$, we use direct count of these levels. Above this value, the density of states is high enough to consider the vibrational level distribution as quasi continuous. As in Ref. 9, we define as "levels" those which consist of all 
states with vibrational energy in the range $E_{v} \pm 10 \mathrm{~cm}^{-1}$. These levels are related to a set of mean values of the vibrational quantum numbers $\left[\bar{v}_{i}\right]$. The fraction of molecules at energy $E_{v}$ which have $v_{i}$ quanta of mode $v_{i}$ is:

$$
F\left(v_{i}, E_{v}\right)=\frac{g\left(E_{v}-v_{i} v_{i}, S=15-d_{i}\right)}{g\left(E_{v}, S=15\right)} \times D
$$

where $D$ is a degeneracy factor equal to 1 for the non-degenerate mode $v_{1}$, and is equal to $v_{i}+1$ for the doubly degenerate mode $v_{2}$ and $\left(v_{i}+\right.$ $1)\left(v_{i}+2\right) / 2$ for the other triply degenerate modes and $d_{i}$ the degeneracy of mode $v_{i}$.

The densities $g\left(E_{v}\right)$ are computed by means of the Whitten and Rabinovitch expression. ${ }^{14}$ For each value of $E_{v}$ there correspond possible integer values of $v_{i}$ from 0 to $x=$ integer $\left(E / v_{i}\right)$ and the mean value of $v_{i}$ is then

$$
\bar{v}_{i}=\sum_{v_{i}=0}^{x} F\left(v_{i}, E_{v}\right) v_{i}
$$

In Ref. 9, it was assumed that if $B^{\prime}$ and $B^{\prime \prime}$ are respectively the rotational constant of the upper and lower states involved in a laser transition, one can neglect the difference $B^{\prime}-B^{\prime \prime}=\Delta B$ as compared to $B^{\prime}$ and $B^{\prime \prime}$. We found it necessary to take into account the linear variation ${ }^{15}$ of the rotational constant $B$ and the Coriolis coupling $\zeta$ as functions of the vibrational energy $E_{v}$, thus expressing empirically, the effective rotational constant as:

$$
B\left(E_{v}\right)=B_{e}-\alpha E_{v} \text { with } \alpha=\alpha_{0}+\beta E_{v}
$$

For each level characterized by a vibrational energy $E_{v}$ and quantum numbers $\left[v_{i}, J\right]$ or $\left[\bar{v}_{i}, J\right]$, there correspond three absorption lines $P, Q$ and $R$ which may coincide with the $\mathrm{CO}_{2}$ laser lines of frequency $\nu_{L}\left(\mathrm{in}^{-1}\right)$ for certain values of $J$, solutions of the following numerical equations 
$P$ branch $v_{L}=v_{o}\left[v_{i}\right]-\left[-0.0538+6.2610^{-4} E_{v}-0.406 \alpha\right] J-\alpha J^{2}$

$Q$ branch $v_{L}=v_{o}\left[v_{i}\right]+\alpha J(J+1)$

$R$ branch $v_{L}=v_{o}\left[v_{i}\right]+\left[0.0538-6.2610^{-4} E_{v}-1.594 \alpha\right] J-\alpha J^{2}$

For a given temperature of $\mathrm{SF}_{6}$, taking into account the vibrational and rotational partition functions, these values of $J$, corresponding to spectral coincidences, determine, within a constant factor, the absorption coefficient $d k\left(\nu_{L}, E_{v}\right)$ of the different $\mathrm{CO}_{2}$ laser lines (of frequency $v_{L}$ ), by each "vibrational level" (of energy $E_{v}$ ). In this band contour model, our only adjustable constants are the linear $\alpha$ and quadratic $\beta$ coefficients of the rotational constant $B$.

\section{Collision model}

We use, as a source of thermal electrons, a beam of argon atoms in Rydberg states which ensure a well controlled energy distribution of these electrons. The collision between the highly excited atoms and the $\mathrm{SF}_{6}$ molecules is a three step process. First, the quasi free electron, with negligible kinetic energy, as compared to the internal energy $E_{v}$ of the colliding $\mathrm{SF}_{6}$ molecule, attaches with a cross-section independent of $E_{v}{ }^{16}$ The negative ion thus produced possesses an internal energy $E_{v}+E A$ where $E A$ is the adiabatic electron affinity of $\mathrm{SF}_{6}$. This ionic core of the excited argon atom suffers Coulomb attraction from the $\mathrm{SF}_{6}^{-}$ion and, in a second step, both ions exchange energy, i.e., part of the internal energy of the $\mathrm{SF}_{6}^{-}$ion can be transferred as kinetic energy to the ionic core. This corresponds to the stabilization process of Zembekov ${ }^{8}$ where, at the end of the collision, the negative ion is left with an internal energy $E_{v}+E A-\Delta E$. Depending on this energy $E_{v^{\prime}}$, in a third step, the negative ion can autodetach with an autoionization rate $\tau_{a}^{-1}\left[E_{v^{\prime}}\right]$, be stabilized (an "infinite" lifetime vs autodetachment) or dissociate with a rate $\tau_{d}^{-1}\left[E_{v^{\prime}}\right]$ (Figure 6).

The dependence of $\tau_{a}, \tau_{d}$ versus the internal energy $E_{v^{\prime}}$ of the negative ion can be estimated by means of RRKM statistical theories ${ }^{17,18}$ or by the inverse Laplace transform of the measured values of negative ion production as a function of temperature. ${ }^{10,19,20}$ The autodetachment lifetime $\tau_{a}$ slowly decreases for $E_{v^{\prime}}$ between 4000 and $8000 \mathrm{~cm}^{-1}$ with a mean value cc. $10 \mu \mathrm{s}$, then drops very small values for $E_{v^{\prime}}$ above $8000 \mathrm{~cm}^{-1}$. The dissociation lifetime $\tau_{d}$ decreases strongly near the threshold energy $E_{d}$ of 


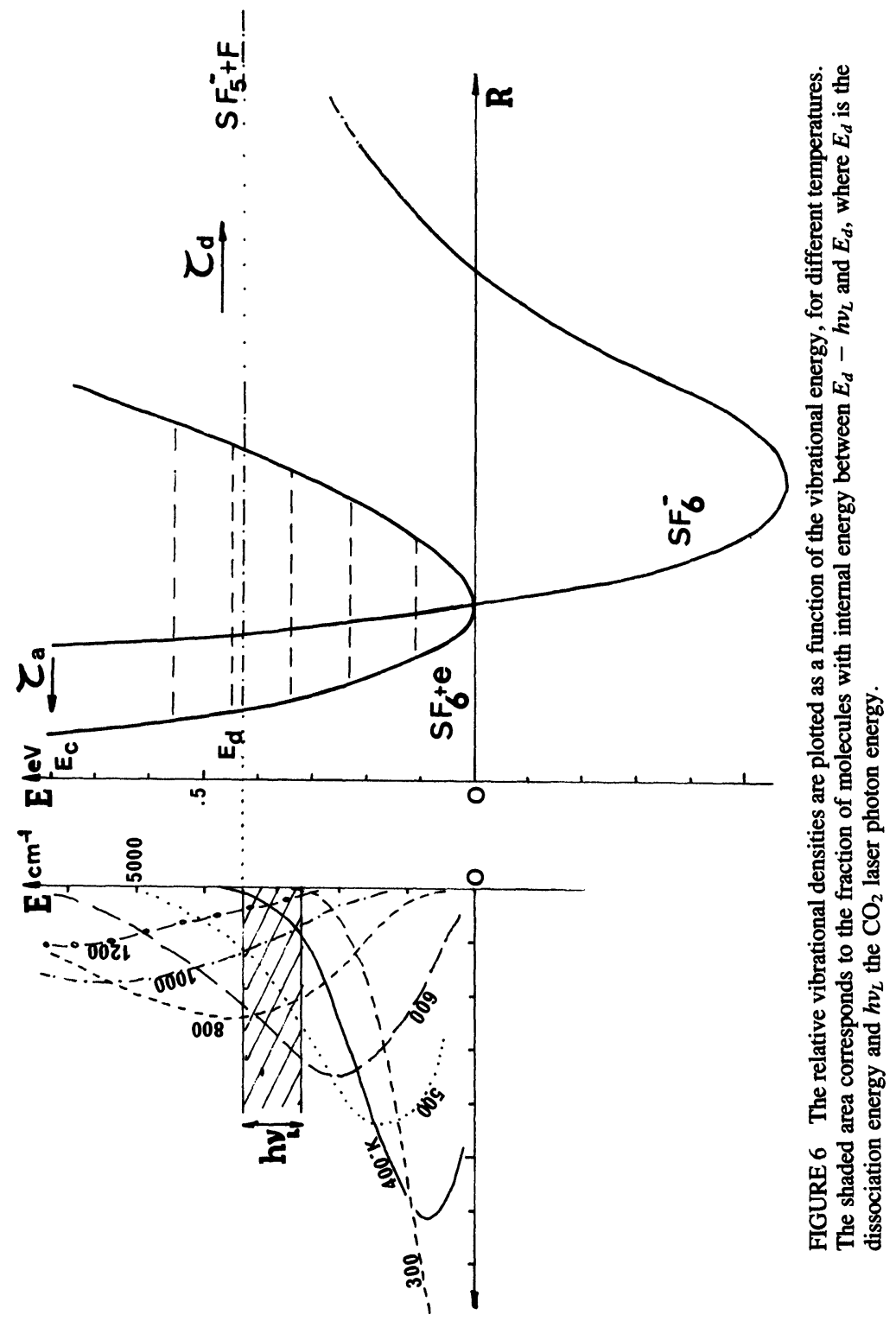


the dissociative reaction $\mathrm{SF}_{6}^{-} \rightarrow \mathrm{SF}_{5}^{-}+\mathrm{F}$, then slowly between 5000 and $8000 \mathrm{~cm}^{-1}$ around a value of $30 \mu \mathrm{s}$.

After the absorption by an $\mathrm{SF}_{6}$ molecule with energy $E_{v}$ of a laser photon of frequency $\nu_{L}$, a negative $\mathrm{SF}_{6}^{-}$ion is produced with an internal energy $E_{v^{\prime \prime}}=E_{v^{\prime}}+h v_{L}=E_{v}+E A-\Delta E+h v_{L}$. During its time of flight $\tau_{p}$ between its creation and its detection this $\mathrm{SF}_{6}^{-}$ion can autodetach or dissociate into $\mathrm{SF}_{5}^{-}$. For each "vibrational level", the variation of the number of detected $\mathrm{SF}_{5}^{-}$ions due to the laser is given by:

$d N\left(\mathrm{SF}_{5}^{-}\right)_{\text {laser }}$

$$
\begin{aligned}
& =C \frac{P}{S} d k\left(v_{L}, E_{v}\right)\left\{\left[1-\exp -\left(\frac{\tau_{p}}{\tau_{a}\left(E_{v^{\prime \prime}}\right)}+\frac{\tau_{p}}{\tau_{d}\left(E_{v^{\prime \prime}}\right)}\right)\right] \frac{\tau_{a}\left(E_{v^{\prime \prime}}\right)}{\tau_{a}\left(E_{v^{\prime \prime}}\right)+\tau_{d}\left(E_{v^{\prime \prime}}\right)}\right. \\
& \left.-\left[1-\exp -\left(\frac{\tau_{p}}{\tau_{a}\left(E_{v^{\prime}}\right)}+\frac{\tau_{p}}{\tau_{d}\left(E_{v^{\prime}}\right)}\right)\right] \frac{\tau_{a}\left(E_{v^{\prime}}\right)}{\tau_{a}\left(E_{v^{\prime}}\right)+\tau_{d}\left(E_{v^{\prime}}\right)}\right\}
\end{aligned}
$$

where $P$ is the laser power, $S$ the laser beam area and $C$ the proportionality constant. $\left(P / S h v_{L}\right) d k\left(v_{L}, E_{v}\right)$ is the number of molecules, in the "vibrational level" $E_{v}$, excited per second and per unit of volume.

The dissociative channel is open to a $\mathrm{SF}_{6}^{-}$ion $\left(E_{v^{\prime}}>E_{d}\right)$ when its parent molecule internal energy $E_{v}$ is above the energy threshold $E_{t h}=E_{d}+$ $\Delta E-h v_{L}$. Thus, the total $\mathrm{SF}_{5}^{-}$ions due to the laser absorption is

$$
N\left(\mathrm{SF}_{5}^{-}\right)_{\text {laser }}=\int_{E_{t h}}^{\infty} d N\left(\mathrm{SF}_{5}^{-}\right)_{\text {laser }}
$$

If we assume that $\tau_{a}$ and $\tau_{d}$ are constant above the energy threshold $E_{v^{\prime}}$ $\geqslant E_{d}+E A$ and that $\tau_{d}$ is infinite for $E_{v^{\prime}}<E_{d}+E A$ then the laser has no effect upon molecules with energy $E_{v} \geqslant E_{d}+\Delta E$ since $\tau\left(E_{v^{\prime \prime}}\right)=$ $\tau\left(E_{v^{\prime}}\right)$. We obtain a simple expression: 


$$
\begin{aligned}
N\left(\mathrm{SF}_{5}^{-}\right) \text {laser }= & \int_{E_{t h}}^{E_{d}+\Delta E} C \frac{P}{S} d k\left(v_{L}, E_{v}\right) \\
& \cdot\left\{\left[1-\exp -\left(\frac{\tau_{p}}{\tau_{a}}+\frac{\tau_{p}}{\tau_{d}}\right)\right] \frac{\tau_{a}}{\tau_{a}+\tau_{d}}\right\} \\
N\left(\mathrm{SF}_{5}^{-}\right) \text {laser }= & \frac{C^{\prime} P}{S} \int_{E_{t h}}^{E_{d}+\Delta E} d k\left(v_{L}, E_{v}\right)
\end{aligned}
$$

The results corresponding to this model are shown in Figure 7 (broken line $E_{c}=0$ ).

In order to take into account the decrease of the branching ratio between dissociation and autodetachment observed in experiments with $\mathrm{SF}_{5}^{-}$and $\mathrm{SF}_{6}^{-},{ }^{10}$ the preceeding model can be improved by assuming that the autodetachment lifetime $\tau_{a}\left(E_{v^{\prime}}\right)$ is constant up to $E_{v^{\prime}}=E A+E_{c}$, and for $E_{v^{\prime}} \geqslant E_{c}+E A . \quad\left(\tau_{a}\left(E_{v^{\prime}}\right)=0\right.$.) This critical value $E_{c}$ is to be determined. With this assumption, we obtain

$$
\begin{aligned}
N\left(\mathrm{SF}_{5}^{-}\right) \text {laser }= & \int_{E_{t h}}^{E_{d}^{+} \Delta E} C \frac{P}{S} d k\left(v_{L}, E_{v}\right) \\
\times & {\left[1-\exp -\left(\frac{\tau_{p}}{\tau_{a}\left(E_{v^{\prime \prime}}\right)}+\frac{\tau_{p}}{\tau_{d}\left(E_{v^{\prime \prime}}\right)}\right)\right] \frac{\tau_{a}\left(E_{v^{\prime \prime}}\right)}{\tau_{a}\left(E_{v^{\prime}}\right)+\tau_{d}\left(E_{v^{\prime \prime}}\right)} } \\
- & \int_{c}^{E_{c}+\Delta E} C \frac{P}{S} d k\left(\nu_{L}, E_{v}\right) \\
& {\left[1-\exp -\left(\frac{\tau_{p}}{\tau_{a}\left(E_{v^{\prime}}\right)}+\frac{\tau_{p}}{\tau_{d}\left(E_{v^{\prime}}\right)}\right)\right] \frac{\tau_{a}\left(E_{v^{\prime}}\right)}{\tau_{a}\left(E_{v^{\prime}}\right)+\tau_{d}\left(E_{v^{\prime}}\right)} }
\end{aligned}
$$

The full line on Figure 7 corresponds to the best fit with $E_{c}=8500 \mathrm{~cm}^{-1}$. 


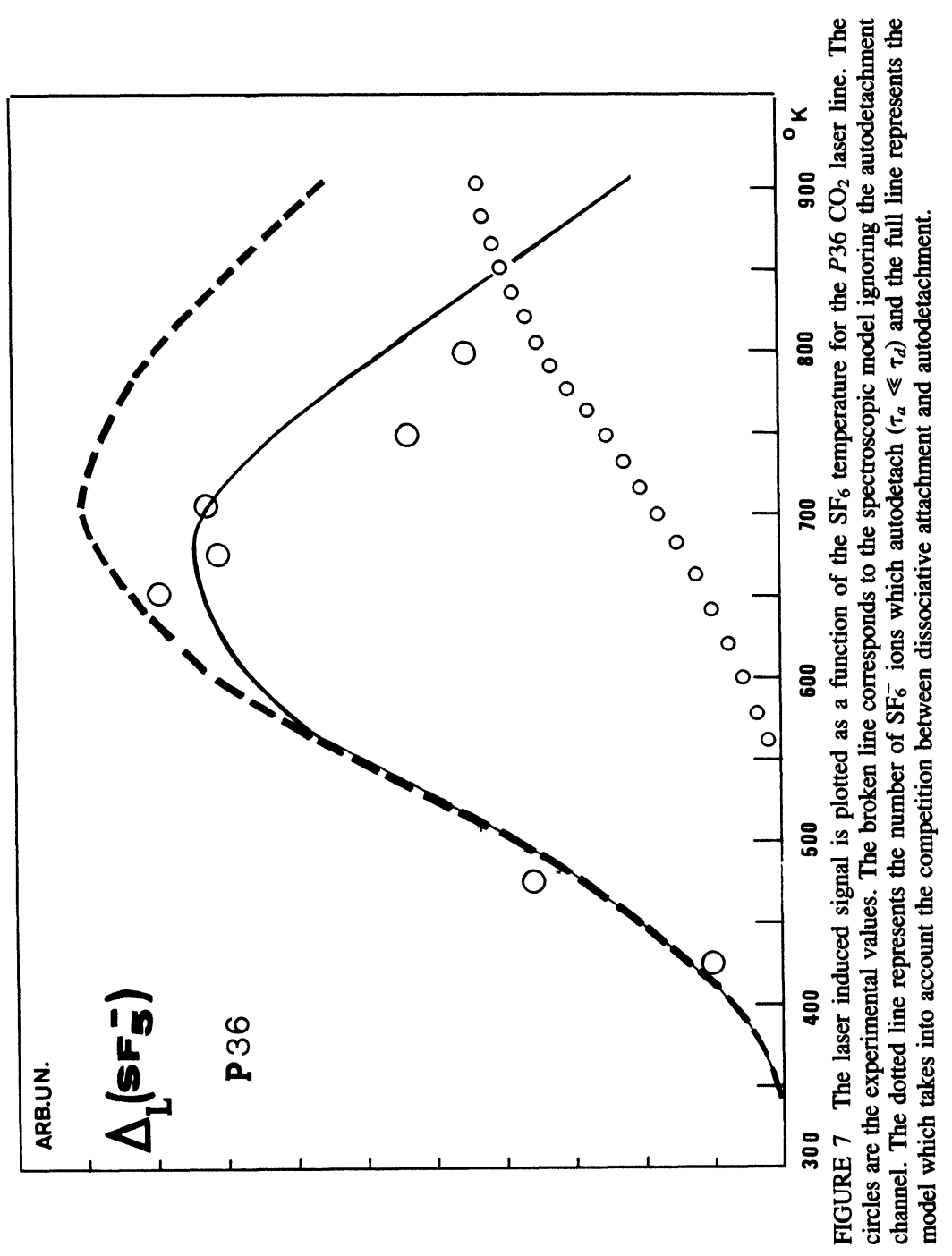




\section{DISCUSSION}

We have measured, as shown in Figures 3 and 4, the rate of production of $\mathrm{SF}_{5}^{-}$ions in the reaction

$$
\begin{aligned}
\mathrm{SF}_{6}\left(E_{v}\right)+h v+\mathrm{Ar}^{* *} \rightarrow \mathrm{SF}_{6}^{*}+\mathrm{Ar}^{* *} \rightarrow & \mathrm{SF}_{6}^{-}\left(E_{v^{\prime}}\right) \\
& +\mathrm{Ar}^{+} \rightarrow \mathrm{SF}_{5}^{-}+\mathrm{F}+\mathrm{Ar}^{+}
\end{aligned}
$$

as a function of the internal energy $E_{v}$ (or temperature) of the parent molecule $\mathrm{SF}_{6}$ above the threshold. Although it has been shown ${ }^{2}$ that for certain values of their internal energy, $\mathrm{SF}_{6}^{-}$ions can absorb $\mathrm{CO}_{2}$ laser radiation, we rule out the possibility of the reaction $\mathrm{SF}_{6}^{-}+h \nu \rightarrow \mathrm{SF}_{5}^{-}$ $+\mathrm{F}$ because the interaction region between the excited neutral $\mathrm{SF}_{6}$ beam and the argon Rydberg atoms are distinct. Moreover, the interaction time between the laser beam and the electrically accelerated $\mathrm{SF}_{6}^{-}$ions would be too short.

We first used the spectroscopic values of Nowack and Lyman to fit our data with the only adjustable parameter $E_{d}$, ignoring the autodetachment level $\left(E_{c} \rightarrow \infty\right)$. The dotted lines in Figure 4 correspond to the best fit with $E_{d}=.53 \mathrm{eV}$. This value is in good agreement with other values determined by several authors ${ }^{8,18,19,23}$ if we assume a mean stabilization energy $\Delta E$ of $.1 \mathrm{eV}$. The dip which appears is due to a lack of overlap between the $P, Q$ and $R$ branches, irrespectively of the value of $E_{d}$. When we introduce the modification of the effective rotational constant as a function of vibrational energy [Eq. (5)], the model correctly predicts the behaviour of the $\mathrm{SF}_{5}^{-}$signal enhancement as a function of temperature for the $\mathrm{CO}_{2}$ laser lines from $P 20$ to $P 26$. For the $\mathrm{CO}_{2}$ laser lines, $P 28$ and above, the model which ignores the autodetachment, predicts too large a signal at high temperatures. When we adjust the value of the energy $E_{c}$ at which the autodetachment channel takes over the dissociative channel, we obtain a reasonably good agreement with the experimental values (Figure 7) with $E_{c}=8500 \mathrm{~cm}^{-1}$.

Using this model, and the spectroscopic values of Table I, we can estimate the relative absorption of the different $\mathrm{CO}_{2}$ laser lines as a function of the excitation energy of the $\mathrm{SF}_{6}$ molecule, for different temperatures. Since, under $1650 \mathrm{~cm}^{-1}$, our model considers the vibrational states as discrete, the absorption spectrum is only represented above this value (this value of $1650 \mathrm{~cm}^{-1}$ is arbitrary and is chosen for computational purposes). 
TABLE I

Numerical values used in the spectroscopic and collision models.

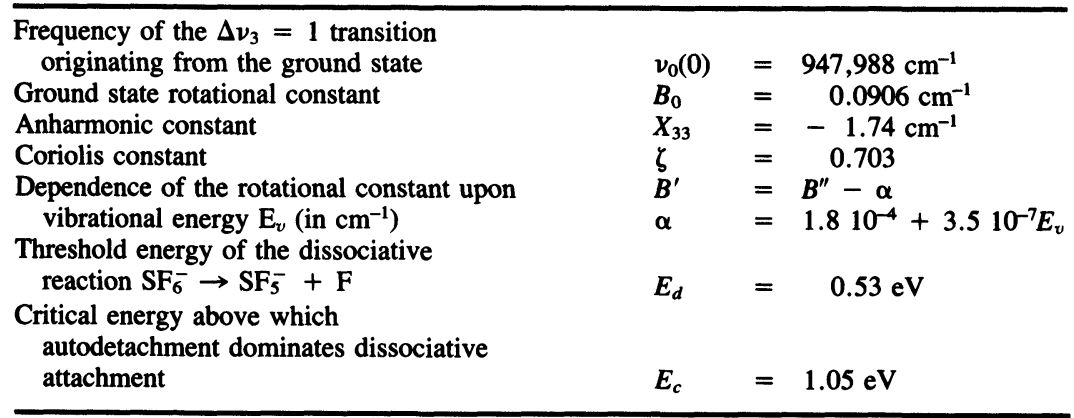

We give only a single example of the discrete absorption spectrum (Figures 8 and 9).

\section{CONCLUSION}

We consider here a reaction between $\mathrm{SF}_{6}$ molecules excited with $3-10$ vibrational quanta, and very low energy electrons. This reaction can be thought of as a probe of the spectroscopic properties of this spectral region of $\mathrm{SF}_{6}$, situated between the well-known fundamental transition of $\nu_{3}$ and the very highly excited vibrational states. This region is, in fact, the one which was considered, in earlier works on isotope separation, ${ }^{1}$ as connecting the resonant $v_{3}$ ladder and the quasi-continuum leading to neutral dissociation.

We found that the Nowack and Lyman ${ }^{9}$ model correctly describes the absorption of the $\mathrm{CO}_{2}$ laser in this excitation region if we take into account a modification of the anharmonicity constant, recently measured, and if we introduce the variation of the effective rotational constant as a function of the vibrational energy.

Our experimental measurements with a well-defined electron energy distribution together with a spectral analysis, confirm the accepted value of $.43 \mathrm{eV}$. for the energy threshold of the dissociative reaction $\mathrm{SF}_{6}^{-} \rightarrow$ $\mathrm{SF}_{5}^{-}+\mathrm{F}$, as opposed to the value of $0.2 \mathrm{eV}$ determined by Chen and 
R. BARBE ET AL.

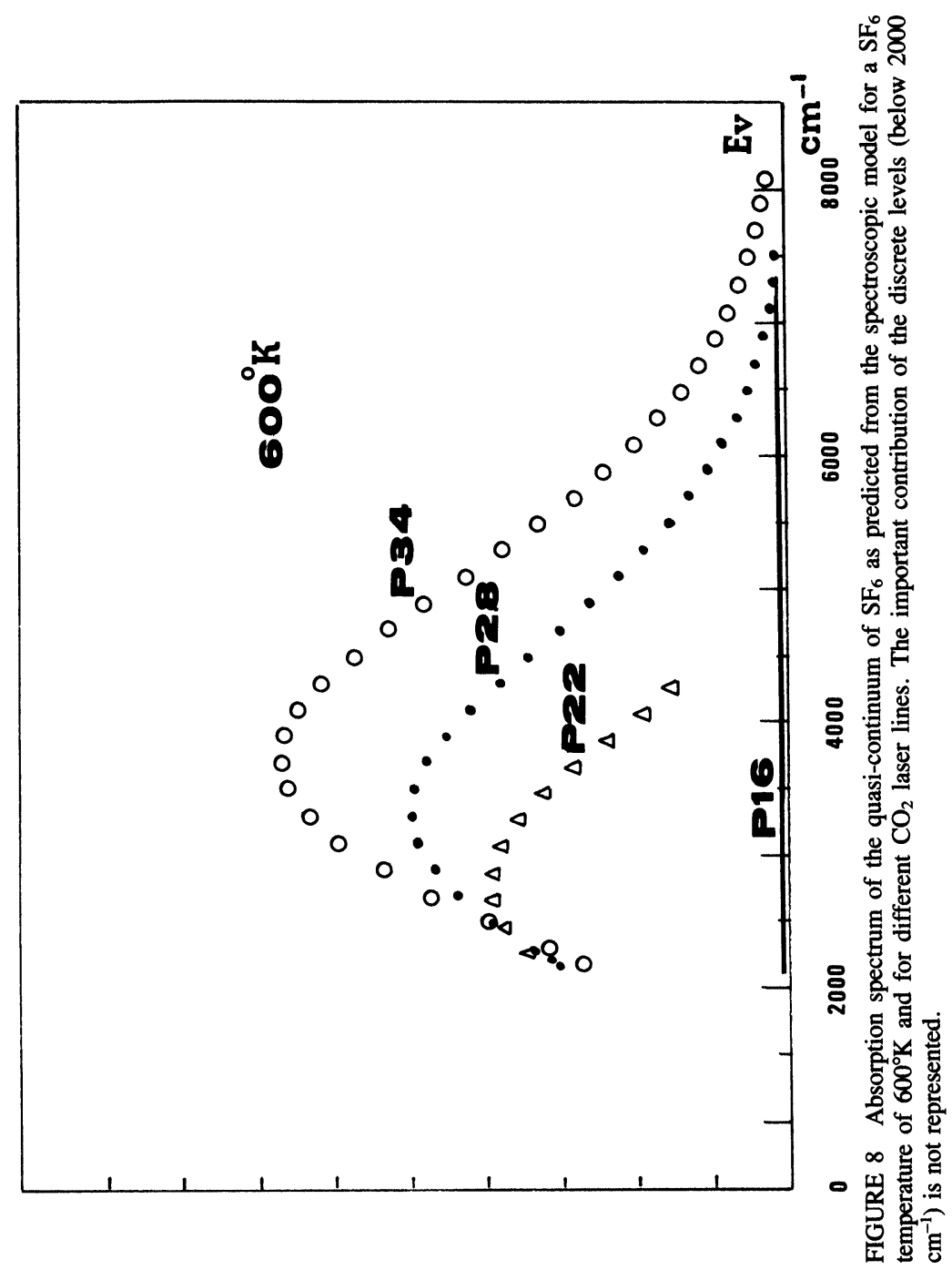




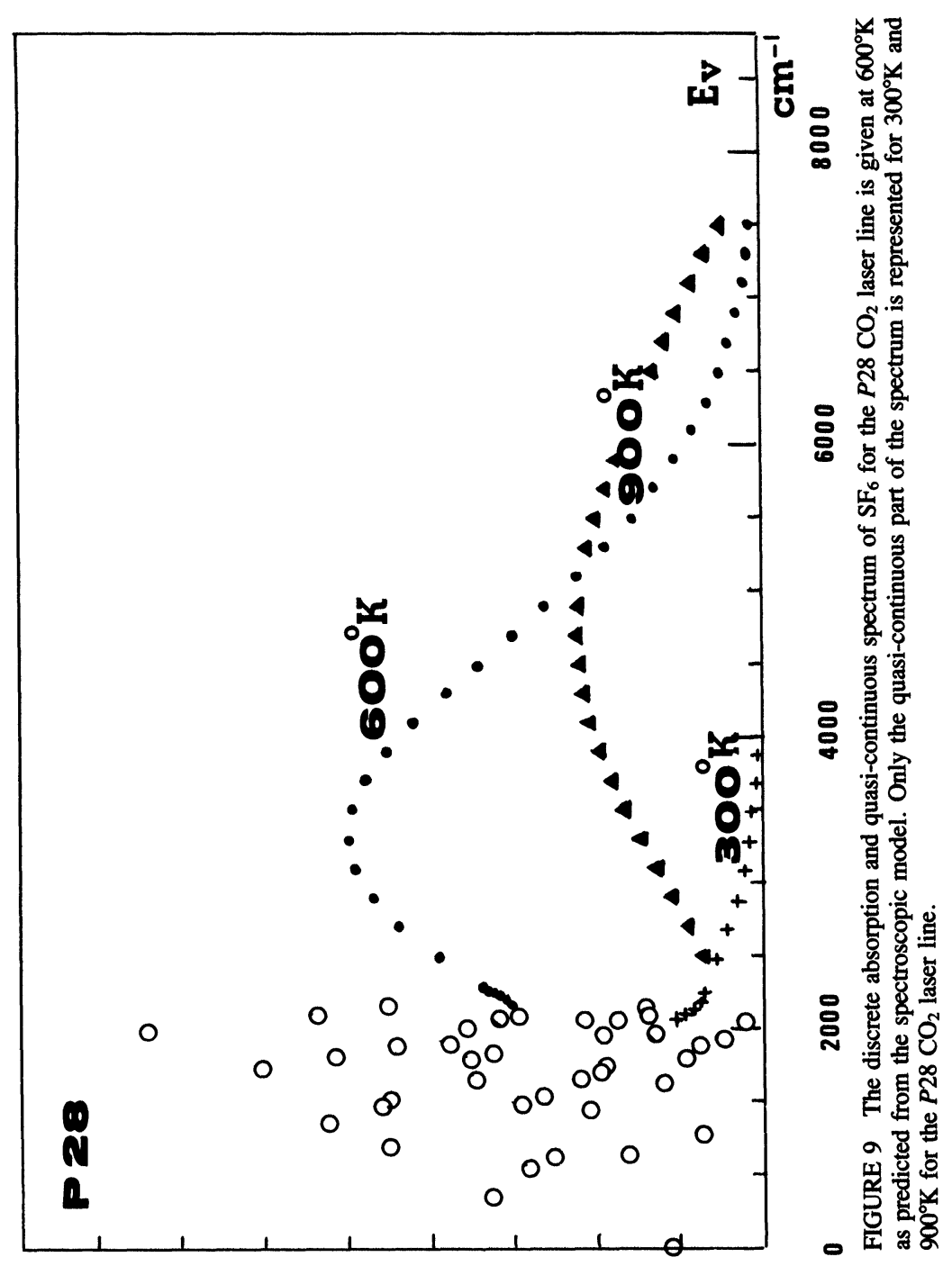


Chantry. This discrepancy is most probably due to the high energy tail of the electron energy distribution used in their experiment. We conclude, from our spectral analysis that the absorption process responsible for the laser enhancement of the dissociative channel is a single photon absorption. We also show that this dissociative process is dominated by an autodetachment channel above a given internal energy of the negative ion that we estimate.

This study of the reaction process which occurs in a collision between an atom in a Rydberg state and a molecule is an example of more general kind of reactions between excited atoms and molecules which can be described as three step processes, the first involving the formation of negative ion by means of a shape or Feshbach resonance, ${ }^{24}$ the second, a stabilization process and the third, competition between several dissociating processes of the negative ion.

\section{References}

1. R. V. Ambartzumian, Y. A. Gorokov, V. S. Letokhov and A. A. Puretskii, JETP Lett. 22, 177 (1975).

2. S. Avrillier and J. P. Schermann, Opt. Comm. 19, 87 (1976).

3. C. L. Chen and P. J. Chantry, J. Chem. Phys. 71, 3897 (1979).

4. R. V. Ambartzumian, Private Communication.

5. R. J. Van Brunt and A. C. Gallagher, Abstract X IPEAC Paris, edited by G. Watel.

6. L. G. Christophorou, Ad. in Electronics and Electr. Phys. 46, 55 (1978).

7. M. Matsuzawa, J. Phys. Soc. Japan 32, 1088 (1972).

8. A. A. Zembekov, Chem. Phys. Lett. 11, 415 (1971).

9. A. V. Nowack and J. L. Lyman, J. Quant. Spectros. Radiat. Transfer. 15, 945 (1975).

10. J. P. Astruc, to be published.

11. Ch. J. Bordé et al., Laser Spectroscopy IV, H. Walther and K. W. Rothe Eds. (Springer Verlag, New York, 1979), p. 142.

12. C. W. Patterson, R. S. Mac Dowell, P. F. Moulton and A. Mooradian, Opt. Lett. 6, 93 (1981).

13. C. W. Patterson, R. J. Krohn and A. S. Pine, Opt. Lett. 6, 39 (1981).

14. E. E. Nikitin, Theory of elementary atomic and molecular processes in gases (Clarendon, Oxford, 1974).

15. G. Herzberg, Molecular Spectra II (Van Nostrand, New York, 1966).

16. D. Spence and G. J. Schultz, J. Chem. Phys. 58, 1800 (1973).

17. C. Klots, Chem. Phys. Lett. 38, 61 (1976).

18. C. Lifshitz and M. Weiss, Chem. Phys. Lett. 15, 266 (1972).

19. M. M. Hubers, J. Los, Chem. Phys. 10, 235 (1975).

20. J. P. Astruc, R. Barbe and J. P. Schermann, J. Phys. B, 12, L377 (1979).

21. P. S. Drzaic and J. I. Brauman, Chem. Phys. Lett. 83, 508 (1981).

22. R. J. Donovan, P. Strachan, H. Stock, R. Parker and J. H. Knox, Dyn. Mass. Spectros. Vol. 4, Ed. D. Price (Heydon, London, 1974).

23. R. J. Compton and P. W. Reinhardt, J. Chem. Phys. 68, 2023 (1978).

24. C. Bottcher and C. V. Sukumar, J. Phys. B, 10, 2853 (1977). 